\title{
On the variational stability of Kähler-Einstein metrics
}

\author{
Xianzhe Dai, Xiaodong Wang and Guofang Wei
}

\begin{abstract}
Using $\operatorname{spin}^{c}$ structure we prove that Kähler-Einstein metrics with non-positive scalar curvature are stable (in the direction of changes in conformal structures) as the critical points of the total scalar curvature functional. Moreover, if all infinitesimal complex deformations of the complex structure are integrable, then the KählerEinstein metric is a local maximal of the Yamabe invariant, and its volume is a local minimum among all metrics with scalar curvature bigger or equal to the scalar curvature of the Kähler-Einstein metric.
\end{abstract}

\section{Introduction}

Stability issue comes up naturally in variational problems. One of the most important geometric variational problems is that of the total scalar curvature functional. Following [1, p. 132], we call an Einstein metric stable if the second variation of the total scalar curvature functional is non-positive in the direction of changes in conformal structures (we have weakened the notion by allowing kernels; see also [20] where stability is defined in terms of local maximum). By the well-known formula, this is to say,

$$
\left\langle\nabla^{*} \nabla h-2 \stackrel{\circ}{R} h, h\right\rangle \geq 0
$$

for any trace-free and divergence-free symmetric 2 -tensor $h$. Here $\stackrel{\circ}{R} h$ denotes the natural action of the curvature tensor on the symmetric tensors [1]. The operator appearing in (1.1) is closely related to the Lichnerowicz

The first author was partially supported by NSF grant \#DMS-0405890 and the third author was partially supported by NSF grant \#DMS-0204187. 
Laplacian $\mathcal{L}_{g}$. Indeed, one has

$$
\mathcal{L}_{g} h=\nabla^{*} \nabla h-2 \stackrel{\circ}{R} h+\text { Ric } \circ h+h \circ \text { Ric. }
$$

The two thus coincide for Ricci flat metrics.

In [12], we studied the stability of compact Ricci flat manifolds. An essential ingredient there is the use of spin structure and parallel spinors. In fact, our result should really be viewed as the stability result for compact Riemannian manifolds with non-zero parallel spinor. By $[14,36]$ this class of manifolds essentially coincides with that of special holonomy, namely, the Calabi-Yau manifolds, hyperKähler manifolds, spin(7) manifolds and $G_{2}$ manifolds.

In this paper, we use $\operatorname{spin}^{c}$ structure to generalize our previous result to manifolds with non-zero parallel $\operatorname{spin}^{c}$ spinor. Since the existence of non-zero parallel spinor implies that the metric is necessarily Ricci flat, our motivation here is to extend our previous method to deal with non-zero scalar curvature and we found $\operatorname{spin}^{c}$ to be a good framework to work with.

Theorem 1.1. If a compact Einstein manifold $(M, g)$ with non-positive scalar curvature admits a non-zero parallel $\mathrm{spin}^{c}$ spinor, then it is stable.

As we mentioned, this generalizes the stability result in [12]. Since a Kähler manifold with its canonical $\operatorname{spin}^{c}$ structure has non-zero parallel $\operatorname{spin}^{c}$ spinors, this implies

Corollary 1.2. A compact Kähler-Einstein manifold with non-positive scalar curvature is stable.

This also follows essentially from Koiso's work $[1,21]$, although it does not seem to have been noticed before. Our approach of using $\operatorname{spin}^{c}$ structure is new and gives more general result. A well-known result in the same direction is for compact Einstein manifolds with negative sectional curvature $[1,19,38]$. In this case the manifold is strictly stable in the sense that the operator $\nabla^{*} \nabla-2 \stackrel{\circ}{R}$ is in fact positive definite. In contrast, there are many Einstein manifolds with positive scalar curvature which are unstable $[9,20]$ (see also [6]).

It turns out that manifolds admitting a non-zero parallel $\operatorname{spin}^{c}$ spinor are more or less classified [26]. Namely a simply connected manifold has a non-zero parallel $\operatorname{spin}^{c}$ spinor if and only if the manifold is the product of a Kähler manifold and a manifold with parallel spinor. Moreover, the 
$\operatorname{spin}^{c}$ structure is the product of the canonical $\operatorname{spin}^{c}$ structure on the Kähler manifold with the spin structure on the other factor.

To prove Theorem 1.1, we derive, for manifolds with non-zero parallel $\operatorname{spin}^{c}$ spinor, a Bochner-type formula relating the operator $\nabla^{*} \nabla-2 \stackrel{\circ}{R}$ to the square of a twisted Dirac operator. The difference, which is expressed in terms of the curvatures, can be shown to be non-negative under our assumption. In fact, we prove that the operator $\nabla^{*} \nabla-2 R$ is positive semi-definite for Kähler manifolds with non-positive Ricci curvature. Our method also proves that the Lichnerowicz Laplacian is positive semi-definite for Kähler manifolds with non-negative Ricci curvature.

The operator $\nabla^{*} \nabla-2 \stackrel{\circ}{R}$ (or the Lichnerowicz Laplacian) seems to have a special penchant for appearing in geometric variational problems. Besides the total scalar curvature functional, or equivalently, the Yamabe functional if one normalizes the volume,

$$
Y(g)=\frac{\int_{M} S_{g} d V_{g}}{\operatorname{Vol}(g)^{1-2 / n}}
$$

there are also the first eigenvalue $\lambda(g)$ of conformal Laplacian considered in [12], the $L^{n / 2}$ norm of scalar curvature [2]

$$
K(g)=\int_{M}\left|S_{g}\right|^{n / 2} d V_{g},
$$

and the Perelman's functionals [9]. Using these functionals, we then deduce a number of interesting consequences.

Theorem 1.3. Let $\left(N, g_{0}, J_{0}\right)$ be a compact Kähler-Einstein manifold with non-positive scalar curvature. Suppose all infinitesimal complex deformations of $J_{0}$ are integrable. Then $g_{0}$ is a local maximum of the Yamabe invariant.

In the case of zero scalar curvature, the integrability condition is automatic by the Bogomolov-Tian-Todorov theorem [5, 33, 35].

Theorem 1.4. Let $\left(N, g_{0}, J_{0}\right)$ be a compact Kähler-Einstein manifold with non-positive scalar curvature. Suppose all infinitesimal complex deformations of $J_{0}$ are integrable. Then any deformation of $g_{0}$ with constant scalar curvature must be Kähler-Einstein.

This generalizes a result of [21] about Einstein deformations. 
Theorem 1.5. Let $\left(N, g_{0}, J_{0}\right)$ be a compact Kähler-Einstein manifold with negative scalar curvature. Suppose all infinitesimal complex deformations of $J_{0}$ are integrable. Then there exists a neighborhood $\mathcal{U}$ of $g_{0}$ in the space of smooth Riemannian metrics on $N$ such that for any metric $g \in \mathcal{U}$ with scalar curvature $S_{g} \geq S_{g_{0}}$

$$
\operatorname{Vol}(N, g) \geq \operatorname{Vol}\left(N, g_{0}\right)
$$

and equality holds if and only if $g$ is a Kähler-Einstein metric with the same scalar curvature.

There are many examples satisfying the assumptions in the theorems above. For example, the hypersurfaces of large enough degree in a complex projective space. In fact, we do not know any examples of Kähler-Einstein manifolds of non-positive scalar curvature which do not satisfy the integrability condition for the complex structure. It is likely that they all satisfy the integrability condition, just as Calabi-Yau manifolds by virtue of the Bogomolov-Tian-Todorov theorem [5,33,35].

The study of the Yamabe constant, also called Schoen's $\sigma$ invariant, has attracted a lot of attention lately, cf. $[7,25]$. This is motivated by a conjecture of Schoen [30], which says that the standard metric for manifolds with constant sectional curvature realizes the Yamabe constant. In other words, the standard metric is a global maximum for the Yamabe invariant (they are called the supreme Einstein metrics in [25]). In view of the results of [3] for real hyperbolic spaces and of [24] for Kähler-Einstein surfaces, it is tempting to conjecture the same for more general class of manifolds such as compact locally symmetric spaces or even Kähler-Einstein manifolds of negative scalar curvature. Unfortunately, this is not true in higher dimensions, as a compact simply connected manifold of dimension greater than or equal to 5 must have non-negative Yamabe constant [27], see also [32] (as some of them will carry metric of positive scalar metric). In [25], an example of simply connected 8-dimensional manifold is given which admit Kähler-Einstein metric with negative scalar curvature, but the Yamabe invariant on it cannot achieve its maximum.

There has been a lot of work recently concerning the stability of Ricci flow $[11,13,31]$, see also [9]. The general question can be phrased as follows. If $g_{0}$ is a metric such that the (renormalized) Ricci flow $g(t)$ starting from $g_{0}$ converges, is it true that the (renormalized) Ricci flow $\tilde{g}(t)$ starting from all metrics $\tilde{g}_{0}$ that are sufficiently close to $g_{0}$ also converges? Using the method of Natasa Sesum [31] (see also [10]), we derive 
Theorem 1.6. Let $\left(N, g_{0}, J_{0}\right)$ be a compact Kähler-Einstein manifold with non-positive scalar curvature. Suppose all infinitesimal complex deformations of $J_{0}$ are integrable. Then the (normalized) Ricci flow starting from any Riemannian metric sufficient close to $g_{0}$ converges exponentially to a Kähler-Einstein metric.

The difference between this theorem and the well-known result for Kähler-Ricci flow on Kähler-Einstein manifolds with non-positive first Chern class [8] is that the Ricci flow here starts with any metric nearby, rather than in a given Kähler class. On the other hand, the result of [8] is a global result in the sense that the initial metric is any metric in a given Kähler class. However, even though we start with any nearby Riemmannian metric, the (normalized) Ricci flow still converges to a Kähler-Einstein metric. This is of course related to Theorem 1.4, see also Theorem 3.6.

This paper is organized as follows. We discuss $\operatorname{spin}^{c}$ parallel spinor and related Bochner-type formula in the next section, and prove the infinitesimal stability result. In Section 3, we discuss the local stability results and applications. In the final section, relevant results from Kodaira-Spencer theory are recalled. We also elaborate more on the examples and make some remarks.

\section{Spin $^{c}$ parallel spinor and a Bochner-type formula}

We now assume $(M, g)$ is a compact Riemannian manifold with a $\operatorname{spin}^{c}$ structure. Thus, $w_{2}(M) \equiv c$, where $c \in H^{2}(M, \mathbb{Z})$ is the canonical class of the $\operatorname{spin}^{c}$ structure. Let $\mathcal{S}^{c} \rightarrow M$ denote the $\operatorname{spin}^{c}$ spinor bundle and $L \rightarrow M$ the complex line bundle with $c_{1}(L)=c$. Then $\mathcal{S}^{c}=\mathcal{S} \otimes L^{1 / 2}$, where the spinor bundle $\mathcal{S}$ may not exist globally; similarly for the square root of the line bundle. An excellent reference on spin geometry is Lawson and Michelsohn [23].

Let $E \rightarrow M$ be a vector bundle with a connection. The curvature is defined as

$$
R_{X Y}=-\nabla_{X} \nabla_{Y}+\nabla_{Y} \nabla_{X}+\nabla_{[X, Y]}
$$

If $M$ is a Riemannian manifold, then for the Levi-Civita connection on $T M$, we have $R(X, Y, Z, W)=\left\langle R_{X Y} Z, W\right\rangle$. We often work with an orthonormal frame $\left\{e_{1}, \ldots, e_{n}\right\}$ and its dual frame $\left\{e^{1}, \ldots, e^{n}\right\}$. Set $R_{i j k l}=R\left(e_{i}, e_{j}, e_{k}, e_{l}\right)$.

The spinor bundle $\mathcal{S}$, which may exist only locally, has a natural connection induced by the Levi-Civita connection on $T M$. For a spinor 
$\sigma$, we have

$$
R_{X Y} \sigma=\frac{1}{4} R\left(X, Y, e_{i}, e_{j}\right) e_{i} e_{j} \cdot \sigma
$$

Given a unitary connection $\nabla^{L}$ on $L$, we then obtain a Clifford connection $\nabla^{c}$ on $\mathcal{S}^{c}$. In fact, $\nabla^{c}=\nabla \otimes 1+1 \otimes \nabla^{L^{1 / 2}}$ is the tensor product connection for $\mathcal{S}^{c}$. Therefore, for a $\operatorname{spin}^{c}$ spinor $\sigma$,

$$
R_{X Y} \sigma=\frac{1}{4} R\left(X, Y, e_{i}, e_{j}\right) e_{i} e_{j} \cdot \sigma-\frac{1}{2} F(X, Y) \sigma .
$$

Here $F$ is the curvature form of $\nabla^{L}$.

If $\sigma_{0}$ is a parallel $\operatorname{spin}^{c}$ spinor, i.e., $\sigma_{0}$ is a section of $\mathcal{S}^{c}$ such that $\nabla_{X}^{c} \sigma_{0}=$ 0 for all $X$, then $R_{X Y} \sigma_{0}=0$. Hence we have

$$
R_{k l i j} e_{i} e_{j} \cdot \sigma_{0}=2 F_{k l} \sigma_{0}
$$

Lemma 2.1. If $\sigma_{0}$ is a parallel spin ${ }^{c}$ spinor, then

$$
R_{k l} e_{l} \cdot \sigma_{0}=F_{k l} e_{l} \cdot \sigma_{0} .
$$

Proof. From (2.4) we have

$$
R_{k l i j} e_{l} e_{i} e_{j} \cdot \sigma_{0}=2 F_{k l} e_{l} \cdot \sigma_{0}
$$

But

$$
\begin{aligned}
R_{k l i j} e_{l} e_{i} e_{j}= & \frac{1}{3} \sum_{l, i, j \text { distinct }}\left(R_{k l i j}+R_{k i j l}+R_{k j l i}\right) e_{l} e_{i} e_{j} \\
& +\sum_{i, j} R_{k j i j} e_{j} e_{i} e_{j}+\sum_{i, j} R_{k i i j} e_{i} e_{i} e_{j} \\
= & 2 R_{k i} e_{i} .
\end{aligned}
$$

Here we have used the symmetries of Riemann curvature tensor, including the first Bianchi identity. Hence,

$$
R_{k l} e_{l} \cdot \sigma_{0}=F_{k l} e_{l} \cdot \sigma_{0}
$$

as claimed.

In the case that the $\operatorname{spin}^{c}$ structure comes from a spin structure, the line bundle $L$ is trivial; consequently $F=0$. Thus Ric $\equiv 0$ for manifolds with non-zero parallel spinor. 
From now on, we assume $M$ has a parallel $\operatorname{spin}^{c}$ spinor $\sigma_{0} \neq 0$, which, without loss of generality, is normalized to be of unit length. We define, as in [12], a linear map $\Phi: S^{2}(M) \rightarrow \mathcal{S}^{c} \otimes T^{*} M$ by

$$
\Phi(h)=h_{i j} e_{i} \cdot \sigma_{0} \otimes e^{j} .
$$

It is easy to check that the definition is independent of the choice of the orthonormal frame $\left\{e_{1}, \ldots, e_{n}\right\}$. The same proof as in [12] again yields

Lemma 2.2. The map $\Phi$ satisfies the following properties.

1. $\operatorname{Re}\langle\Phi(h), \Phi(\tilde{h})\rangle=\langle h, \tilde{h}\rangle$,

2. $\nabla_{X} \Phi(h)=\Phi\left(\nabla_{X} h\right)$.

Here Re denotes the real part.

The following interesting Bochner-type formula plays an important role here.

Lemma 2.3. Let $h$ be a symmetric 2-tensor on $M$. Then

$$
\mathcal{D}^{*} \mathcal{D} \Phi(h)=\Phi\left(\nabla^{*} \nabla h-2 \stackrel{\circ}{R} h-h \circ F+\operatorname{Ric} \circ h\right) .
$$

Here $(h \circ F)_{i j}=h_{i p} F_{p j}=-h_{i p} F_{j p}$ and $(\operatorname{Ric} \circ h)_{i j}=R_{i p} h_{j p}$.

Remark 2.4. Note that here we have implicitly extended our map $\Phi$ to general (non-symmetric) 2-tensors with complex coefficients.

Proof of Lemma 2.3. Choose an orthonormal frame $\left\{e_{1}, \ldots, e_{n}\right\}$ near a point $p$ such that $\nabla e_{i}=0$ at $p$. We compute at $p$, using Lemma 2.2 and the Ricci identity,

$$
\begin{aligned}
\mathcal{D}^{*} \mathcal{D} \Phi(h) & =\nabla_{e_{k}} \nabla_{e_{l}} h\left(e_{i}, e_{j}\right) e_{k} e_{l} e_{i} \cdot \sigma_{0} \otimes e^{j} \\
& =-\nabla_{e_{k}} \nabla_{e_{k}} h\left(e_{i}, e_{j}\right) e_{i} \cdot \sigma_{0} \otimes e^{j}-\frac{1}{2} R_{e_{k} e_{l}} h\left(e_{i}, e_{j}\right) e_{k} e_{l} e_{i} \cdot \sigma_{0} \otimes e^{j} \\
& =\Phi\left(\nabla^{*} \nabla h\right)+\frac{1}{2} R_{k l j p} h_{i p} e_{k} e_{l} e_{i} \cdot \sigma_{0} \otimes e^{j}+\frac{1}{2} R_{k l i p} h_{p j} e_{k} e_{l} e_{i} \cdot \sigma_{0} \otimes e^{j}
\end{aligned}
$$

By using twice the Clifford relation $e_{i} e_{j}+e_{j} e_{i}=-2 \delta_{i j}$ we have

$$
\begin{aligned}
\frac{1}{2} R_{k l j p} h_{i p} e_{k} e_{l} e_{i} \cdot \sigma_{0} & =\frac{1}{2} R_{k l j p} h_{i p} e_{i} e_{k} e_{l} \cdot \sigma_{0}+R_{k l j p} h_{k p} e_{l} \cdot \sigma_{0}-R_{k l j p} h_{l p} e_{k} \cdot \sigma_{0} \\
& =F_{j p} h_{i p} e_{i} \cdot \sigma_{0}-2(\stackrel{\circ}{R} h)_{k j} e_{k} \cdot \sigma_{0} .
\end{aligned}
$$


Here the last equality uses (2.4). On the other hand,

$$
\frac{1}{2} R_{k l i p} h_{p j} e_{k} e_{l} e_{i} \cdot \sigma_{0}=\frac{1}{2} h_{p j}\left(R_{k l i p} e_{k} e_{l} e_{i} \cdot \sigma_{0}\right)=R_{l p} h_{p j} e_{l} \cdot \sigma_{0}
$$

Putting these equations together we obtain our lemma.

Once again, when the $\operatorname{spin}^{c}$ structure comes from a spin structure, the formula above becomes

$$
\mathcal{D}^{*} \mathcal{D} \Phi(h)=\Phi\left(\nabla^{*} \nabla h-2 \stackrel{\circ}{R} h\right)
$$

which recovers a formula of [37], see also [12]. By Lemma 2.2, the stability result follows in this case [12].

The existence of a parallel $\operatorname{spin}^{c}$ spinor on a compact simply connected manifold implies that the manifold is the product of a Kähler manifold with a manifold with parallel spinor [26]. Moreover, the $\operatorname{spin}^{c}$ structure is the product of the canonical $\operatorname{spin}^{c}$ structure on the Kähler manifold with the spin structure on the other factor.

We now assume that $(M, g)$ is a compact Kähler manifold of real dimension $n=2 m$. Let $J$ be the parallel almost complex structure and $\omega=g(J \cdot, \cdot)$ the Kähler form. The complexified tangent bundle decomposes as

$$
T M \otimes \mathbb{C}=T^{1,0} M \oplus T^{0,1} M .
$$

The canonical $\operatorname{spin}^{c}$ structure is given by the anti-canonical line bundle $L=$ $K^{-1}=\Lambda^{m}\left(T^{1,0}(M)\right)$. It has a canonical holomorphic connection induced from the Levi-Civita connection and the curvature form $F=-\sqrt{-1} \rho$, where $\rho=\operatorname{Ric}(J \cdot, \cdot)$ is the Ricci form.

The spinor bundle $\mathcal{S}^{c}(M)=\mathcal{S}_{+}^{c}(M) \bigoplus \mathcal{S}_{-}^{c}(M)$ with

$$
\begin{aligned}
& \mathcal{S}_{+}^{c}(M)=\bigoplus_{k \text { even }} \Lambda^{0, k}(M), \\
& \mathcal{S}_{-}^{c}(M)=\bigoplus_{k \text { odd }} \Lambda^{0, k}(M) .
\end{aligned}
$$

The Clifford multiplication is defined by

$$
\left.v \cdot=\sqrt{2}\left(v^{0,1} \wedge-v^{0,1}\right\lrcorner\right)
$$

Here $\left.v^{0,1}\right\lrcorner$ denotes the contraction using the Hermitian metric. The parallel spinor $\sigma \in C^{\infty}\left(\mathcal{S}_{+}^{c}(M)\right)$ can be taken as the function which is identically 1 . 
We now choose our orthonormal basis $e_{1}, \ldots, e_{2 m}$ such that $e_{m+i}=J e_{i}$. By a slight abuse of notation, we denote $e_{i}=e_{m+i}=J e_{i}$. And similarly the index $\bar{i}$ denote $m+i$. Hence, with $\sigma=1$ being the parallel $\operatorname{spin}^{c}$ spinor, we have

$$
\begin{aligned}
& \left\langle\sigma, e_{i} e_{j} \cdot \sigma\right\rangle=-\delta_{i j}, \quad\left\langle\sigma, e_{i}^{-} e_{j} \cdot \sigma\right\rangle=-\delta_{i j}, \\
& \left\langle\sigma, e_{i} e_{j} \cdot \sigma\right\rangle=-\sqrt{-1} \delta_{i j}, \quad\left\langle\sigma, e_{i} e_{\bar{j}} \cdot \sigma\right\rangle=\sqrt{-1} \delta_{i j} .
\end{aligned}
$$

Now we compute

$$
\begin{aligned}
-\langle\Phi(h \circ F), \Phi(h)\rangle= & \sum_{i, j, k, l, p=1}^{2 m} F_{j p} h_{i p} h_{k l}\left\langle e_{i} \cdot \sigma \otimes e^{j}, e_{k} \cdot \sigma \otimes e^{l}\right\rangle \\
= & -\sum_{i, j, k, p=1}^{2 m} F_{j p} h_{i p} h_{k j}\left\langle\sigma, e_{i} e_{k} \cdot \sigma\right\rangle \\
= & \sum_{j, p=1}^{2 m} \sum_{i=1}^{m} F_{j p} h_{i p} h_{i j}-\sum_{j, p=1}^{2 m} \sum_{i=1}^{m} F_{j p} h_{i p} h_{\overline{i j}}(\sqrt{-1}) \\
& -\sum_{j, p=1}^{2 m} \sum_{i=1}^{m} F_{j p} h_{\overline{i p}} h_{i j}(-\sqrt{-1})+\sum_{j, p=1}^{2 m} \sum_{i=1}^{m} F_{j p} h_{\overline{i p}} h_{\overline{i j}} .
\end{aligned}
$$

As the curvature of a unitary connection on a line bundle, $F$ is purely imaginary. Hence taking the real part (and using the skew symmetry) yields:

$$
-\operatorname{Re}\langle\Phi(h \circ F), \Phi(h)\rangle=-2 \sqrt{-1} \sum_{j, p=1}^{2 m} \sum_{i=1}^{m} F_{j p} h_{i p} h_{i j} .
$$

Similarly,

$$
\operatorname{Re}\langle\Phi(\operatorname{Ric} \circ h), \Phi(h)\rangle=\sum_{i, j, p=1}^{2 m} R_{i p} h_{p j} h_{i j}
$$

We are now ready to prove

Theorem 2.5. If $\left(M, g_{0}\right)$ is a compact Kähler manifold with non-positive Ricci curvature, then $\nabla^{*} \nabla h-2 \stackrel{\circ}{R} h$ is positive semi-definite on $S^{2}(M)$. 
That is,

$$
\left\langle\nabla^{*} \nabla h-2 \stackrel{\circ}{R} h, h\right\rangle \geq\langle\mathcal{D} \Phi(h), \mathcal{D} \Phi(h)\rangle \geq 0,
$$

for any $h \in S^{2}(M)$. Moreover, in the case of negative Ricci curvature, $\nabla^{*} \nabla h-2 \stackrel{\circ}{R} h=0$ if and only if $\mathcal{D} \Phi(h)=0$ and $h$ is skew-Hermitian.

Proof. Since $\langle\mathcal{D} \Phi(h), \mathcal{D} \Phi(h)\rangle \geq 0$, we have, by Lemmas 2.3 and 2.2 ,

$$
\begin{aligned}
0 & \leq\left\langle\mathcal{D}^{*} \mathcal{D} \Phi(h), \Phi(h)\right\rangle \\
& =\operatorname{Re}\left\langle\Phi\left(\nabla^{*} \nabla h-2 \stackrel{\circ}{R} h-h \circ F+\operatorname{Ric} \circ h\right), \Phi(h)\right\rangle \\
& =\left\langle\nabla^{*} \nabla h-2 \stackrel{\circ}{R} h, h\right\rangle-\operatorname{Re}\langle\Phi(h \circ F), \Phi(h)\rangle+\operatorname{Re}\langle\Phi(\operatorname{Ric} \circ h), \Phi(h)\rangle .
\end{aligned}
$$

That is,

$$
\begin{aligned}
\left\langle\nabla^{*} \nabla h-2 \stackrel{\circ}{R} h, h\right\rangle= & \langle\mathcal{D} \Phi(h), \mathcal{D} \Phi(h)\rangle-[\operatorname{Re}\langle\Phi(\operatorname{Ric} \circ h), \Phi(h)\rangle \\
& -\operatorname{Re}\langle\Phi(h \circ F), \Phi(h)\rangle] .
\end{aligned}
$$

For $L=K^{-1}$, as we remarked earlier, the curvature form $F=-\sqrt{-1} \rho$, where $\rho$ is the Ricci form. Since $g$ is Kähler, there is an orthonormal basis $e_{1}, \ldots, e_{2 m}$ such that $e_{m+i}=J e_{i}(1 \leq i \leq m)$ and the Ricci curvature is diagonal in this basis, i.e., $R_{i j} c_{i} \delta_{i j}(1 \leq i, j \leq 2 m)$ with $c_{i}=c_{m+i}$. Now

$$
\rho\left(e_{i}, e_{j}\right)=\operatorname{Ric}\left(J e_{i}, e_{j}\right)= \begin{cases}c_{i} \delta_{m+i, j}, & 1 \leq i \leq m \\ -c_{i} \delta_{m-i, j}, & m+1 \leq i \leq 2 m .\end{cases}
$$

It follows then from (2.9) and (2.10) that

$$
\begin{gathered}
-\operatorname{Re}\langle\Phi(h \circ F), \Phi(h)\rangle=-2 \sum_{j=1}^{m} \sum_{i=1}^{m} c_{j}\left(h_{i \bar{j}} h_{\overline{i j}}-h_{i j} h_{\overline{i j}}\right) . \\
\operatorname{Re}\langle\Phi(\operatorname{Ric} \circ h), \Phi(h)\rangle=\sum_{i, j=1}^{2 m} c_{i} h_{i j}^{2} .
\end{gathered}
$$

Hence,

$$
\left\langle\nabla^{*} \nabla h-2 \stackrel{\circ}{R} h, h\right\rangle \geq-\left[\sum_{i, j=1}^{2 m} c_{i} h_{i j}^{2}-2 \sum_{j=1}^{m} \sum_{i=1}^{m} c_{i}\left(h_{i \bar{j}} h_{\overline{i j}}-h_{i j} h_{\overline{i j}}\right)\right] .
$$

When $c_{i} \leq 0$ the right-hand side is non-negative by the Cauchy-Schwarz inequality. 
If $c_{i}<0$, then $\nabla^{*} \nabla h-2 \stackrel{\circ}{R} h=0$ if and only if $\mathcal{D} \Phi(h)=0$ and

$$
h_{i j}=-h_{\bar{i} \bar{j}}, \quad h_{i \bar{j}}=h_{\bar{i} j} .
$$

That is, $h(J X, J Y)=-h(X, Y)$. It follows that $h$ is skew Hermitian.

Similarly, we have

Theorem 2.6. If $\left(M, g_{0}\right)$ is a compact Kähler manifold with non-negative Ricci curvature, then the Lichnerowicz Laplacian is positive semi-definite on $S^{2}(M)$. That is,

$$
\left\langle\mathcal{L}_{g} h, h\right\rangle \geq\langle\mathcal{D} \Phi(h), \mathcal{D} \Phi(h)\rangle \geq 0,
$$

for any $h \in S^{2}(M)$. Moreover, in the case positive Ricci curvature, $\mathcal{L}_{g} h=0$ if and only if $\mathcal{D} \Phi(h)=0$ and $h$ is Hermitian.

Proof. The Lichnerowicz Laplacian is

$$
\mathcal{L}_{g} h=\nabla^{*} \nabla h-2 \stackrel{\circ}{R} h+\text { Ric } \circ h+h \circ \text { Ric } .
$$

The same proof as above now goes through for $\mathcal{L}_{g}$.

Note that the above computation in the case of Kähler-Einstein manifold with Einstein constant $c$ yields the following interesting BochnerLichnerowics-Weitzenbock formula:

$$
\langle\mathcal{D} \Phi(h), \mathcal{D} \Phi(h)\rangle=\left\langle\nabla^{*} \nabla h-2 \stackrel{\circ}{R} h, h\right\rangle+2 c\left\langle h_{H}, h_{H}\right\rangle,
$$

where $h_{H}$ denotes the Hermitian part of $h$. This unifies the two Weitzenbock formulas in [1, p. 362].

\section{Local stability of Käher-Einstein metrics}

Theorem 2.5 says that for a Kähler-Einstein $\left(N, g_{0}\right)$ with non-positive scalar curvature the operator

$$
\nabla^{*} \nabla h-2 \stackrel{\circ}{R} h
$$

is semi-positive definite on symmetric 2-tensors. A natural and important question is to identify the kernal space

$$
W_{g_{0}}=\left\{h \mid \operatorname{tr}_{g_{0}} h=0, \delta h=0, \nabla^{*} \nabla h-2 \stackrel{\circ}{R} h=0\right\}
$$


on the space of transverse traceless symmetric 2-tensors. This is just the infinitesimal Einstein deformation space studied in [21]. The case $c=0$ is essentially a Calabi-Yau manifold, which has been studied with other manifolds admitting parallel spinors in our previous paper [12]. We now focus on the case $c<0$ using our approach.

By the proof of Theorem 2.5

$$
W_{g_{0}}=\left\{h \mid \operatorname{tr}_{g_{0}} h=0, \delta h=0, h(J, J)=-h, \mathcal{D} \Phi(h)=0\right\}
$$

As before we choose our orthonormal basis $e_{1}, \ldots, e_{2 m}$ such that $e_{m+i}=J e_{i}$.

Now for $1 \leq i \leq m$ set

$$
X_{i}=\frac{e_{i}-\sqrt{-1} J e_{i}}{\sqrt{2}}, \quad \bar{X}_{i}=\frac{e_{i}+\sqrt{-1} J e_{i}}{\sqrt{2}} .
$$

Then $\left\{X_{1}, \ldots, X_{m}\right\}$ is a local unitary frame for $T^{1,0} M$ and let its dual frame be $\left\{\theta^{1}, \ldots, \theta^{m}\right\}$. As $h$ is skew-Hermitian we have

$$
h\left(X_{i}, \bar{X}_{j}\right)=h\left(\bar{X}_{j}, X_{i}\right)=0
$$

By straightforward computation we have for $h \in W=W_{g_{0}}$,

$$
\Phi(h)=h\left(\bar{X}_{i}, \bar{X}_{j}\right) \bar{\theta}^{i} \otimes \bar{\theta}^{j} .
$$

This can be identified with

$$
\Psi(h)=h\left(\bar{X}_{i}, \bar{X}_{j}\right) \bar{\theta}^{i} \otimes X_{j} \in \wedge^{0,1}(\Theta),
$$

where $\Theta$ is the holomorphic tangent bundle. We compute

$$
\begin{aligned}
\mathcal{D} \Phi(h)= & \sum_{k=1}^{m}\left(\nabla_{e_{k}} h\left(\bar{X}_{i}, \bar{X}_{j}\right) e_{k} \cdot \bar{\theta}^{i} \otimes \bar{\theta}^{j}+\nabla_{e_{\bar{k}}} h\left(\bar{X}_{i}, \bar{X}_{j}\right) e_{\bar{k}} \cdot \bar{\theta}^{i} \otimes \bar{\theta}^{j}\right) \\
= & \sum_{k=1}^{m}\left(\nabla_{e_{k}} h\left(\bar{X}_{i}, \bar{X}_{j}\right)\left(\bar{\theta}^{k} \wedge \bar{\theta}^{i}-\delta_{i k}\right) \otimes \bar{\theta}^{j}+\nabla_{e_{\bar{k}}} h\left(\bar{X}_{i}, \bar{X}_{j}\right)\left(\sqrt{-1} \bar{\theta}^{k} \wedge \bar{\theta}^{i}\right.\right. \\
& \left.\left.-\sqrt{-1} d_{i k}\right) \otimes \bar{\theta}^{j}\right) \\
= & \sqrt{2} \sum_{k=1}^{m}\left(\nabla_{\bar{X}_{k}} h\left(\bar{X}_{i}, \bar{X}_{j}\right) \bar{\theta}^{k} \wedge \bar{\theta}^{i} \otimes \bar{\theta}^{j}-\nabla_{X_{k}} h\left(\bar{X}_{k}, \bar{X}_{j}\right) \bar{\theta}^{j}\right) .
\end{aligned}
$$

With $\Phi(h)$ identified as $\Psi(h) \in \wedge^{0,1}(\Theta)$, the above calculation shows that the Dirac operator is then $\sqrt{2}\left(\bar{\partial}-\bar{\partial}^{*}\right)$ on $\Psi(h)$. Therefore $\mathcal{D} \Phi(h)=0$ if and 
only if $\Psi(h)$ is harmonic. On the other hand

$$
\begin{aligned}
\delta h & =\sum_{k=1}^{m}\left(\nabla_{e_{k}} h\left(e_{k}, \cdot\right)+\nabla_{e_{\bar{k}}} h\left(e_{\bar{k}}, \cdot\right)\right) \\
& =\sum_{k=1}^{m}\left(\nabla_{X_{k}} h\left(\bar{X}_{k}, \cdot\right)+\nabla_{\bar{X}_{k}} h\left(X_{k}, \cdot\right)\right) \\
& =\sum_{j, k=1}^{m}\left(\nabla_{X_{k}} h\left(\bar{X}_{k}, \bar{X}_{j}\right) \bar{\theta}^{j}+\nabla_{\bar{X}_{k}} h\left(X_{k}, X_{j}\right) \theta^{j}\right)
\end{aligned}
$$

where in the last step we used (3.4). This shows that $\delta h=0$ automatically holds if $\Psi(h)$ is harmonic. Therefore we have an injective homomorphism

$$
\Psi: W_{g_{0}} \rightarrow H^{1}(N, \Theta)
$$

The image obviously consists of symmetric infinitesmial complex deformations. To show that $\Psi$ is, in fact, onto we need to show all infinitesimal complex deformations are symmetric. For this purpose we need a digression.

Let $N$ be a Kähler manifold with Kähler metric $\omega=\sqrt{-1} g_{i j} d z^{i} \wedge d \bar{z}^{j}$. Given $\Psi=a \frac{i}{j} d \bar{z}^{j} \otimes \frac{\partial}{\partial z^{i}} \in \wedge^{0,1}\left(T^{1,0} N\right)$, we can consider the $(0,2)$-form

$$
\psi=g_{i \bar{l}} a_{\bar{j}}^{i} d \bar{z}^{j} \wedge d \bar{z}^{l}
$$

Remark 3.1. One can work equally well with a local unitary frame $\left\{X_{1}, \ldots, X_{m}\right\}$ and its dual frames, but it seems that the calculations are easier with local coordinates.

We calculate

$$
\begin{aligned}
\bar{\partial} \Psi & =\frac{\partial a_{\bar{l}}^{i}}{\partial \bar{z}^{j}} d \bar{z}^{j} \wedge d \bar{z}^{l} \otimes \frac{\partial}{\partial z^{i}} \\
\bar{\partial}^{*} \Psi & \left.=-g^{k \bar{l}} \frac{\partial}{\partial \bar{z}^{l}}\right\rfloor \nabla_{\frac{\partial}{\partial z^{k}} \Psi} \\
& =-g^{k \bar{l}}\left(\frac{\partial a_{\bar{l}}^{i}}{\partial z^{k}}+\Gamma_{k p}^{i} a_{\bar{l}}^{p}\right) \frac{\partial}{\partial z^{i}}
\end{aligned}
$$

Suppose now that $\Psi$ is harmonic, i.e., $\bar{\partial} \Psi=0, \bar{\partial}^{*} \Psi=0$. Then we have

$$
\frac{\partial a_{\bar{l}}^{i}}{\partial \bar{z}^{j}}=\frac{\partial a_{\bar{j}}^{i}}{\partial \bar{z}^{l}}
$$


and

$$
g^{k \bar{l}}\left(\frac{\partial a_{\bar{l}}^{i}}{\partial z^{k}}+\Gamma_{k p}^{i} a_{\bar{l}}^{p}\right)=0
$$

Thus

$$
\bar{\partial} \psi=\left(g_{i \bar{l}} \frac{\partial a_{\bar{j}}^{i}}{\partial \bar{z}^{q}}+a \frac{i}{\bar{j}} \frac{\partial g_{i \bar{l}}}{\partial \bar{z}^{q}}\right) d \bar{z}^{q} \wedge d \bar{z}^{j} \wedge d \bar{z}^{l}=0,
$$

here we used (3.7) and the fact that $\frac{\partial g_{i \bar{l}}}{\partial \bar{z}^{q}}$ is symmetric in $l$ and $q$.

We calculate

$$
\begin{aligned}
\bar{\partial}^{*} \psi & \left.=-g^{k \bar{l}} \frac{\partial}{\partial \bar{z}^{l}}\right\rfloor \nabla_{\partial / \partial z^{k}} \psi \\
& \left.=-g^{k \bar{l}} \frac{\partial}{\partial \bar{z}^{l}}\right\rfloor\left[\left(g_{i \bar{q}} \frac{\partial a_{\bar{j}}^{i}}{\partial z^{k}}+\frac{\partial g_{i \bar{q}}}{\partial z^{k}} a_{\bar{j}}^{i}\right) d \bar{z}^{j} \wedge d \bar{z}^{q}\right] \\
& =-g^{k \bar{l}}\left[\left(g_{i \bar{q}} \frac{\partial a_{\bar{l}}^{i}}{\partial z^{k}}+\frac{\partial g_{i \bar{q}}}{\partial z^{k}} a_{\bar{l}}^{i}\right) d \bar{z}^{q}-\left(g_{i \bar{l}} \frac{\partial a_{\bar{j}}^{i}}{\partial z^{k}}+\frac{\partial g_{k \bar{l}}}{\partial z^{i}} a_{\bar{j}}^{i}\right) d \bar{z}^{j}\right] \\
& =-g_{i \bar{q}} g^{k \bar{l}}\left(\frac{\partial a_{\bar{l}}^{i}}{\partial z^{k}}+\Gamma_{k p}^{i} a_{\bar{l}}^{p}\right) d \bar{z}^{q}+\left(\frac{\partial a_{\bar{j}}^{i}}{\partial z^{i}}+\frac{\partial \log \operatorname{det} G}{\partial z^{i}} a_{\bar{j}}^{i}\right) d \bar{z}^{j} \\
& =\left(\frac{\partial a_{\bar{j}}^{i}}{\partial z^{i}}+\frac{\partial \log \operatorname{det} G}{\partial z^{i}} a_{\bar{j}}^{i}\right) d \bar{z}^{j}
\end{aligned}
$$

where in the last step we used (3.8). Therefore

$$
\begin{aligned}
\overline{\partial \partial}^{*} \psi & \left(\frac{\partial^{2} a \frac{i}{j}}{\partial z^{i} \partial \bar{z}^{l}}+\frac{\partial \log \operatorname{det} G}{\partial z^{i}} \frac{\partial a_{\bar{j}}^{i}}{\partial \bar{z}^{l}}+\frac{\partial^{2} \log \operatorname{det} G}{\partial z^{i} \partial \bar{z}^{l}} a \frac{i}{j}\right) d \bar{z}^{l} \wedge d \bar{z}^{j} \\
& =-\frac{\partial^{2} \log \operatorname{det} G}{\partial z^{i} \partial \bar{z}^{l}} a \frac{i}{j} d \bar{z}^{j} \wedge d \bar{z}^{l}
\end{aligned}
$$

where in the last step we used the fact that the first two coefficients are symmetric in $j$ and $l$ by (3.7). To summarize we have

$$
\overline{\partial \partial}^{*} \psi=R_{i \bar{l}} a \frac{i}{j} d \bar{z}^{j} \wedge d \bar{z}^{l} .
$$

Theorem 3.2. Let $\left(N, \omega_{0}\right)$ be a compact Kähler-Einstein manifold with negative scalar curvature. Suppose $\Psi=a_{\frac{j}{j}}^{i} d \bar{z}^{j} \otimes \frac{\partial}{\partial z^{i}} \in \wedge^{0,1}\left(T^{1,0} N\right)$ is harmonic. Then $\psi=g_{i \bar{l}} a_{\bar{j}}^{i} d \bar{z}^{j} \wedge d \bar{z}^{l}=0$, i.e., $g_{i \bar{l}} a_{\bar{j}}^{i}$ is symmetric in $i$ and $j$. 
Proof. By the assumption we have $R_{i \bar{j}}=c g_{i \bar{j}}$ with $c<0$. By $(3.9)$

$$
\overline{\partial \partial}^{*} \psi=c \psi
$$

Therefore

$$
c \int_{N}|\psi|^{2}=\int_{N}\left\langle\overline{\partial \partial}^{*} \psi, \psi\right\rangle=\int_{N}\left|\bar{\partial}^{*} \psi\right|^{2}
$$

Since $c<0$ we must have $\psi=0$.

Remark 3.3. In the Ricci flat case the above calculation shows that for a harmonic $\Psi \in H^{1}(N, \Theta)$ the corresponding $(0,2)$-form $\psi$ is indeed harmonic. Conversely one can show that a harmonic $(0,2)$-form $\psi$ gives rise to a skew-symmetric infinitesimal complex deformation. Therefore the space of skew-symmetric infinitesimal complex deformations can be identified as the space of holomorphic $(2,0)$-forms.

Remark 3.4. The argument in Besse [1, 12.96] showing there are no skew-symmetric infinitesimal complex deformations on a Kähler-Einstein manifold with negative scalar curvature is incorrect. In particular the claim that skew-symmetric infinitesimal complex deformations are in one-to-one correspondence with holomorphic 2 -forms is not true. In fact there are compact complex hyperbolic surfaces $N$ with $H^{1}(N, \Theta)=0$ and $h^{2,0}(N) \neq 0$.

As a corollary of Theorem 3.2 we now have a clear understanding of the kernel $W_{g_{0}}$ of $(3.2)$.

Theorem 3.5. Let $\left(N, g_{0}, J_{0}\right)$ be a Kähler-Einstein manifold with negative scalar curvature. Then $\Psi: W_{g_{0}} \rightarrow H^{1}(N, \Theta)$ is an isomorphism.

By the Kodaira-Spencer theory, $H^{1}(N, \Theta)$ is the space of infinitesimal complex deformations on $N$. In general these infinitesimal deformations may not be integrable. But if they are integrable, then the premoduli space of complex structures on $N$ is an manifold near $J$, with $H^{1}(N, \Theta)$ as the tangent space (see the next section for more discussion). In this case we can deduce various local results which we now explain. The argument is by now standard, see [12] and Besson-Courtois-Gallot [2] where same type of results are established for Einstein manifolds with negative sectional curvature.

We consider two well-known functionals, in addition to the first eigenvalue $\lambda(g)$ considered in [12]. For a compact manifold $(M, g)$ of 
dimension $n$

$$
K(g)=\int_{M}\left|S_{g}\right|^{n / 2} d V_{g}
$$

and

$$
Y(g)=\frac{\int_{M} S_{g} d V_{g}}{\operatorname{Vol}(g)^{1-2 / n}}
$$

Theorem 3.6. Let $\left(N, g_{0}, J_{0}\right)$ be a compact Kähler-Einstein manifold with negative scalar curvature. Suppose all infinitesimal complex deformations of $J_{0}$ are integrable. Then there exists a neighborhood $\mathcal{U}$ of $g_{0}$ in the space of smooth Riemannian metrics on $N$ such that

$$
\forall g \in \mathcal{U} \quad K(g) \geq K\left(g_{0}\right)
$$

and equality holds if and only if $g$ is a Kähler-Einstein metric with negative scalar curvature. Moreover, all Einstein metrics in $\mathcal{U}$ are Kähler-Einstein with negative scalar curvature.

Since all infinitesimal complex deformations of $J_{0}$ are integrable, the premoduli space of complex structures on $N$ is an manifold near $J_{0}$, with $H^{1}(N, \Theta)$ as the tangent space. By the uniqueness of Kähler-Einstein metric with negative scalar curvature and the implicit function theorem, the moduli space $\mathcal{E}$ of Kähler-Einstein metrics is an orbifold near $g_{0}$, with $W_{g_{0}} \cong H^{1}(N, \Theta)$ as the tangent space.

Both functionals $K$ and $Y$ are scaling invariant, therefore we can restrict ourselves to the space of Riemannian metric of volume 1, denoted by $\mathcal{M}$. By Ebin's slice theorem, there is a real submanifold $\mathcal{S}$ containing $g_{0}$, which is a slice for the action of the diffeomorphism group on $\mathcal{M}$. The tangent space

$$
T_{g_{0}} \mathcal{S}=\left\{h \mid \delta_{g_{0}} h=0, \int_{N} \operatorname{tr}_{g_{0}} h d V_{g_{0}}=0\right\} .
$$

Let $\mathcal{C} \subset \mathcal{S}$ be the submanifold of constant scalar curvatures metrics.

We need the following simple lemma from [2].

Lemma 3.7. Let $g$ be a metric with scalar curvature a negative constant and $g^{\prime}$ a metric conformal to $g$. Then $K\left(g^{\prime}\right) \geq K(g)$ and equality holds if and only if $g^{\prime}=g$. 
By this Lemma and the solution of the Yamabe problem, we only need to prove $g_{0}$ is a local minimum for the functional $K$ on $\mathcal{C}$. So it suffices to work on $\mathcal{C}$. It is easy to see

$$
T_{g_{0}} \mathrm{C}=\left\{h \mid \delta_{g_{0}} h=0, \operatorname{tr}_{g_{0}} h=0\right\}
$$

Restricted on $\mathcal{C}$ and in a neighborhood of $g_{0}$, the functional $K$ becomes $K(g)=\left|S_{g}\right|^{m}=\left(-S_{g}\right)^{m}$. Therefore to prove that $g_{0}$ is a local minimum for $K$ on $\mathrm{C}$ is equivalent to prove that $Y$ has a local maximum at $g_{0}$ on $\mathrm{C}$. It is well known that $g_{0}$ is a critical point for $Y$ and its Hessian at $g_{0}$ is given by

$$
D^{2} Y(h, h)=-\frac{1}{2} \int_{N}\left\langle\nabla^{*} \nabla h-2 \stackrel{\circ}{R} h, h\right\rangle
$$

$\mathcal{C}$ contains the finite dimensional submanifold $\mathcal{E}$ of Kähler-Einstein metrics, with tangent space $W_{g_{0}} \cong H^{1}(N, \Theta)$. For any $g \in \mathcal{E}$, let $J$ be the associated complex structure. We have

$$
\rho_{g}=\frac{S_{g}}{2 m} \omega_{g}
$$

where $\omega_{g}$ is the associated Kähler form and $\rho_{g}$ the Ricci form. Therefore

$$
Y(g)=-4 m \pi\left(\frac{\left(-C_{1}(N, J)\right)^{m}[N]}{m !}\right)^{1 / m}
$$

where $C_{1}(N, J)$ is the first Chern class of $(N, J)$. Thus $Y$ is constant on $\mathcal{E}$. Moreover, we have proved that $D^{2} Y$ is negative definite on its normal bundle. Therefore there is a possibly smaller neighborhood of $\mathcal{E} \subset \mathcal{C}$, still denoted by $\mathcal{U}$, such that

$$
\forall g \in \mathcal{U}-\mathcal{E}, \quad Y(g)<Y\left(g_{0}\right)
$$

This proves Theorem 3.6.

We could have used $\lambda(g)$ instead of $Y(g)$ for the proof, as in [12].

Theorem 3.8. Let $\left(N, g_{0}, J_{0}\right)$ be a compact Kähler-Einstein manifold with negative scalar curvature. Suppose all infinitesimal complex deformations of $J_{0}$ are integrable. Then there exists a neighborhood $\mathcal{U}$ of $g_{0}$ in the space of smooth Riemannian metrics on $N$ such that for any metric $g \in \mathcal{U}$ with 
scalar curvature $S_{g} \geq S_{g_{0}}$

$$
\operatorname{Vol}(N, g) \geq \operatorname{Vol}\left(N, g_{0}\right)
$$

and equality holds if and only if $g$ is a Kähler-Einstein metric with the same scalar curvature.

Remark 3.9. Though only a local result, it is quite remarkable to have volume comparison under a lower bound for the scalar curvature. The scalar curvature is a very weak geometric quantity and its effect on a general Riemannian manifold $(M, g)$ of dimension $n$ can only be detect infinitesimally by the following expansion for the volume of a geodesic ball $B(p, r)$

$$
\operatorname{Vol}(B(p, r))=\omega_{n} r^{n}\left(1-\frac{S_{g}(p)}{6(n+2)} r^{2}+\mathrm{O}\left(r^{3}\right)\right) \quad \text { as } r \rightarrow 0
$$

Proof. We take the same $\mathcal{U}$ in Theorem 3.6. Then $\forall g \in \mathcal{U}$ with $S_{g} \geq S_{g_{0}}$, we have $\left|S_{g}\right|^{m} \leq\left|S_{g_{0}}\right|^{m}$ since $S_{g}<0$. Therefore $K(g) \leq\left|S_{g_{0}}\right|^{m} \operatorname{Vol}(N, g)$ while $K\left(g_{0}\right)=\left|S_{g_{0}}\right|^{m} \operatorname{Vol}\left(N, g_{0}\right)$. The result then follows from Theorem 3.6.

Theorem 3.6 has another interesting interpretation. Recall that the Yamabe invariant of a compact Riemannian manifold $(M, g)$ of dimension $n$ is

$$
\mu(g)=\inf _{f \in C^{\infty}(M), f>0} Y\left(f^{(n-2) / 4} g\right)
$$

and it is a conformal invariant. The Yamabe number of $M$ is defined as

$$
\sigma(M)=\sup _{g} \mu(g)
$$

We can now reformulate Theorem 3.6 as follows.

Theorem 3.10. Let $\left(N, g_{0}, J_{0}\right)$ be a compact Kähler-Einstein manifold with negative scalar curvature. Suppose all infinitesimal complex deformations of $J_{0}$ are integrable. Then $g_{0}$ is a local maximum of the Yamabe invariant.

We end with a few remarks. In [30] Schoen made the following conjecture.

Conjecture 3.11. Let $\left(M, g_{0}\right)$ be a compact hyperbolic manifold. Then $\sigma(M)$ is achieved by $g_{0}$ and only by $g_{0}$. In other words

$$
\forall g \quad \mu(g) \leq \mu\left(g_{0}\right)
$$

and equality holds if and only if $g$ is conformal to $g_{0}$. 
It is also reasonable to make the same conjecture for other compact locally symmetric spaces with negative sectional curvature. In complex dimension 2 there have been some remarkable results proved by LeBrun using Seiberg-Witten invariants. For example, he proved that on any compact Kähler-Einstein surface with negative scalar curvature the Yamabe number is achieved by the Kähler-Einstein metric. See [24,25].

In view of LeBrun's result and Theorem 3.10 it is tempting to extend the conjecture to Kähler-Einstein manifolds $\left(N, g_{0}\right)$ with negative scalar curvature in higher dimensions, namely that $g_{0}$ should be a global maximum of the Yamabe invariant provided all its infinitesimal complex deformations are integrable. But in general this is not true. In fact any compact and simply connected manifold $M$ of dimension at least 5 has $\sigma(M) \geq 0$. This is trivial if $M$ admits a metric of positive scalar curvature [32]. Otherwise it is proved by Petean [27].

Another intriguing question is whether Theorems 3.6 and 3.10 are still true if there are non-integrable complex deformations. Then we have infinitesimal Käher-Einstein deformations which cannot be integrated to Käher-Einstein metrics, but they may be integrated to Einstein metrics which are not Kähler.

\section{Kodaira-Spencer theory}

The deformation theory of complex structures was introduced by KodairaSpencer in their seminal work [16-18]. This deep theory has played and still plays significant role in the theory of complex manifolds. The relation between Kodaira-Spencer theory and the deformation of Einstein metrics has been studied by Koiso [21]. We review some relevant facts here in this section and discuss some examples in more detail.

Let $M$ be a compact complex manifold and $\Theta$ the (sheaf of germs of the) holomorphic tangent bundle of $M$. According to the Kodaira-Spencer theory, the infinitesimal complex deformations are described by the cohomology group $H^{1}(M, \Theta)$. For our purpose, we are interested in the integrability of infinitesimal complex deformations. Namely, when does every infinitesimal deformation actually arise from a deformation of complex structures? Let us recall first the so-called theorem of existence in the Kodaira-Spencer theory [15, Theorem 5.6].

Theorem 4.1 (Kodaira-Spencer). Let $M$ be a compact complex manifold. If $H^{2}(M, \Theta)=0$, then there is a complex analytic family with base $B$, 
$0 \in B \subset \mathbb{C}^{m}$ such that the fiber at 0 is $M$ and the Kodaira-Spencer map at 0 is an isomorphism from $T_{0} B$ onto $H^{1}(M, \Theta)$.

Recall that the Kodaira-Spencer map for a differentiable family of compact complex manifolds assigns a tangent vector of the base to the infinitesimal deformation along that direction. Thus the condition $H^{2}(M, \Theta)=0$ implies that all infinitesimal complex deformations are integrable.

We now discuss some examples from [15], where the reader is referred to for complete detail.

Example 4.2. Blowups of $\mathbb{C P}^{2}$. For $M=\mathbb{C P}^{2} \# k \overline{\mathbb{C P}^{2}}$ and $k \geq 5$, one has $H^{0}(M, \Theta)=H^{2}(M, \Theta)=0$. Hence, all infintesimal deformations are integrable. Incidentally, for $k \leq 4, H^{1}(M, \Theta)=0$. Therefore the complex structure is rigid in these cases.

It is well known that there exists Kähler-Einstein metrics on $M$ if and only if $3 \leq k \leq 8$ by Tian's work [34]. However, these Kähler-Einstein metrics have positive scalar curvature. Hence our results do not apply. In fact, other than $\mathbb{C P}^{2}$ itself, these are unstable, [9].

Example 4.3. Surfaces of arbitrary degree. For a non-singular surface $M$ of degree $h$ in $\mathbb{C P}^{3}$, one has

$$
\operatorname{dim} H^{2}(M, \Theta)=\frac{1}{2}(h-2)(h-3)(h-5) .
$$

Thus, $H^{2}(M, \Theta)=0$ for $h=2,3,5$. Since $c_{1}(M)=(4-h) H$ where $H$ is the hyperplane class, $M$ has Kähler-Einstein metrics with negative scalar curvature if $h \geq 5$, by the Calabi-Aubin-Yau theorem. Hence our results apply to the non-singular surface of degree 5 .

As one can see here, in general, the condition $H^{2}(M, \Theta)=0$, which guarantees the integrability of all infinitesimal complex deformations, is very restrictive. Indeed, there are many examples which do not satisfy this condition but still, all their infinitesimal complex deformations are integrable. In fact, understanding the reason behind this is one of the motivations for Kodaira and Spencer.

From the Kodaira-Spencer theory, if an infinitesimal complex deformation $\theta \in H^{1}(M, \Theta)$ is integrable, then

$$
[\theta, \theta]=0
$$

This is in fact the first order obstruction. One thus expects that there should be non-integrable infinitesimal deformations. However, the cohomology 
group $H^{1}(M, \Theta)$ turns out to be surprisingly difficult to compute. And in the many examples where it can be computed, the infinitesimal deformations turn out to be integrable.

Recall that a complex analytic family of compact complex manifolds is said to be effective (or minimal) if its Kodaira-Spencer map is injective. It is called a complete (or versal) family if every other (sufficiently small) family of deformations can be induced from this family via pullback of a holomorphic map. Now whenever there is an effective complete family with base $B \ni 0$ a domain in $\mathbb{C}^{m}$ such that the fiber at 0 is $M$, Kodaira-Spencer defines the number of moduli $m(M)=m$ to be the dimension of the base. Then the question of whether all infinitesimal deformations are integrable can be reinterpreted as when the equality $m(M)=\operatorname{dim} H^{1}(M, \Theta)$ holds, of which Kodaira-Spencer refers as the fundamental guiding question in the Kodaira-Spencer theory.

By the theorem of completeness in the Kodaira-Spencer theory [15, Theorem 6.1], which says that a complex analytic family of compact complex manifolds with surjective Kodaira-Spencer map is complete, the complex analytic family in Theorem 4.1 is an effective complete family with base dimension $\operatorname{dim} H^{1}(M, \Theta)$.

More generally, if $M$ is a compact complex manifold for which there is a complex analytic family of deformations whose Kodaira-Spencer map is surjective, then all infinitesimal deformations are integrable. This is the case where many examples can be found. (We will see the converse in a moment.)

Example 4.4. Hypersurfaces in $\mathbb{C P}^{n}$. If $M$ is a hypersurface in $\mathbb{C P}^{n}$ of degree $d$, one can construct a complex analytic family of deformations of $M$ by varying the coefficients of the defining equation of $M$. This family has surjective Kodaira-Spencer map. There are many examples in this class which admits Kähler-Einstein metrics with negative scalar curvature. Let $N \subset \mathbf{C P}^{m+1}$ be a smooth algebraic hypersurface of degree $d>m+2$. Then the 1st Chern class $c_{1}(N)<0$. By the theorem of Calabi-Aubin-Yau, there is a Kähler-Einstein metric with negative scalar curvature $g_{0}$ on $N$. It is shown in [15, p. 219] that

$$
\operatorname{dim} H^{1}(N, \Theta)=\left(\begin{array}{c}
m+1+d \\
d
\end{array}\right)-(m+2)^{2} .
$$

Going back to the question of existence, without any assumptions, there is the Kuranishi theorem [22]. 
Theorem 4.5 (Kuranishi). For any compact complex manifold $M$, there exists a complete complex analytic family with base $B, 0 \in B$ such that the fiber at 0 is $M$. Moreover $B$ is a complex analytic subset of $\mathbb{C}^{m}$, where $m=\operatorname{dim} H^{1}(M, \Theta)$, defined by $l$ holomorphic equations, with $l=$ $\operatorname{dim} H^{2}(M, \Theta)$.

It can be deduced from Kuranishi's theorem that if every infinitesimal complex deformations are integrable, then the Kuranishi family above is a complex analytic family whose Kodaira-Spencer map is an isomorphism (and hence surjective). Thus, our integrability assumption is equivalent to the existence of complex analytic family of deformations whose KodairaSpencer map is surjective.

As pointed out by Koiso [21], the existence of such a family has significant implication for the moduli space of (Kähler-)Einstein metrics.

Theorem 4.6. Let $M$ be a compact complex manifold and $(g, J)$ be a Kähler-Einstein structure on $M$. Assume that the complex structure $J$ belongs to a complex analytic family of complex structures with surjective Kodaira-Spencer map. Moreover, if the scalar curvature is positive, assume further that there is no non-zero Hermitian infinitesimal Einstein deformations and also no non-zero holomorphic vector field. Then the local premoduli space of Einstein metrics around $g$ is a manifold with tangent space at $g$ the space of infinitesimal Einstein deformations. Moreover, any Einstein metric in it is Kähler (with respect to some complex structure).

It should be pointed out that there are indeed examples of compact complex manifolds with non-integrable infinitesimal complex deformations [15, p. 319]. However, we do not know any examples of Kähler-Einstein manifolds with negative scalar curvature which does not satisfy the integrability condition. In view of the Bogmolov-Tian-Todorov theorem $[5,33,35]$ in the Calabi-Yau case, we have the following very interesting question.

Question: Is it true that on any compact Kähler-Einstein manifolds with negative scalar curvature, the universal deformation space of complex structures is smooth?

\section{Acknowledgment}

The authors wish to thank Rick Schoen for stimulating discussions and encouragement. 


\section{References}

[1] Arthur L. Besse, Einstein manifolds, Springer-Verlag, Berlin, 1987.

[2] G. Besson, G. Courtois and S. Gallot, Volume et entropie minimale des espaces localement symétriques, Invent. math. 103 (1991), 417-445.

[3] G. Besson, G. Courtois and S. Gallot, Entropies et rigidits des espaces localement symtriques de courbure strictement ngative, Geom. Funct. Anal. 5(5) (1995), 731-799.

[4] D. Blair, The "total scalar curvature" as a symplectic invariant and related results, Proceedings of the 3rd Congress of Geometry (Thessaloniki, 1991), Aristotle University Thessaloniki, Thessaloniki, 1992, 79-83.

[5] F.A. Bogomolov, Hamiltonian Kähler manifolds, Dolk. Akad. Nauk SSSR 243(5) (1978), 1101-1104.

[6] C. Böhm, Unstable Einstein metrics, Math. Z. 250(2) (2005), 279-286.

[7] H. Bray and A. Neves, Classification of prime 3-manifolds with Yamabe invariant greater than $\mathbb{R P}^{3}$, Ann. of Math. (2) 159(1) (2004), 407-424.

[8] H. Cao, Deformation of Kähler metrics to Kähler-Einstein metrics on compact Kähler manifolds, Invent. Math. 81(2) (1985), 359-372.

[9] Huai-Dong Cao, Richard S. Hamilton and Tom Ilmanen, Gaussian densities and stability for some Ricci solitons, Preprint arXiv: math.DG/0404165.

[10] J. Cheeger and G. Tian, On the cone structure at infinity of Ricci flat manifolds with Euclidean volume growth and quadratic curvature decay, Invent. Math. 118(3) (1994), 493-571.

[11] X. Chen, On the lower bound of energy functional $E_{1}$ (I)- a stability theorem on the Kaehler Ricci flow, J. Geom. Anal. 16(1) (2006), 23-38.

[12] X. Dai, G. Wei and X. Wang, On the stability of Riemannian manifold with parallel spinors, Invent. Math. 161(1) (2005), 151-176.

[13] C. Guenther, J. Isenberg and D. Knopf, Stability of the Ricci flow at Ricci-flat metrics, Comm. Anal. Geom. 10(4) (2002), 741-777.

[14] N. Hitchin, Harmonic spinors, Adv. in Math. 14 (1974), 1-55.

[15] K. Kodaira, Complex manifolds and deformations of complex structures, Fundamental principles of Mathematical Sciences, SpringerVerlag, New York, 1986, 283. 
[16] K. Kodaira and D. Spencer, On deformations of complex analytic structures, I-II, Ann. Math. 67 (1958), 328-466.

[17] K. Kodaira and D. Spencer, A theorem of completeness for complex analytic fibre spaces, Acta Math. 100 (1958), 281-294.

[18] K. Kodaira and D. Spencer, On deformations of complex analytic structures, III, Ann. Math. 71 (1960), 43-76.

[19] N. Koiso, A decomposition of the space $\mathcal{M}$ of Riemannian metrics on a manifold, Osaka J. Math. 16(2) (1979), 423-429.

[20] N. Koiso, Rigidity and stability of Einstein metrics - the case of compact symmetric spaces, Osaka J. Math. 17 (1980), 51-73.

[21] N. Koiso, Einstein metrics and complex structures, Invent. Math. 73 (1983), 71-106.

[22] M. Kuranishi, On the locally complete families of complex analytic structures, Ann. Math. 75 (1962), 536-577.

[23] H. Blaine Lawson, Jr. and Marie-Louise Michelsohn, Spin geometry, Princeton University Press, Princeton, NJ, 1989.

[24] LeBrun, Einstein metrics and Mostow rigidity, Math. Res. Lett. 2 (1995), 1-8.

[25] C. LeBrun, Einstein metrics and the Yamabe problem, Trends in Mathematical Physics, American Mathematical Society, 13, Providence, RI, 1999, 353-376.

[26] A. Moroianu, Parallel and Killing Spinors on Spinc Manifolds, Commun. Math. Phys. 187 (1997), 417-427.

[27] J. Petean, The Yamabe invariant of simply connected manifolds, J. Reine Angew. Math. 523 (2000), 225-231.

[28] G. Perelman, The entropy formula for the Ricci flow and its geometric applications, Preprint, arXiv: math.DG/0211159.

[29] Richard M. Schoen, Conformal deformation of a Riemannian metric to constant scalar curvature, J. Differential Geom. 20(2) (1984), 479-495.

[30] Richard M. Schoen, Variational theory for the total scalar curvature functional for Riemannian metrics and related topics, Topics in calculus 
of variations (Montecatini Terme, 1987), Springer, Berlin, 1989, 120-154.

[31] N. Sesum, Linear and dynamical stability of Ricci flat metrics, Duke Math. J. 133 (1) (2006), 1-26.

[32] S. Stolz, Simply connected manifolds of positive scalar curvature, Ann. of Math. 136 (1992), 511-540.

[33] G. Tian, Smoothness of the universal deformation space of compact Calabi-Yau manifolds and its Petersson-Weil metric, Mathematical aspects of string theory, Adv. Ser. Math. Phys., World Scientific, Singapore, 1987, 629-646.

[34] G. Tian, Kähler-Einstein metrics with positive scalar curvature, Invent. Math. 130 (1) (1997), 1-37.

[35] A. Todorov, The Weil-Petersson geometry of the moduli space of $\mathrm{SU}(n \geq 3)$ (Calabi-Yau) manifolds. I, Comm. Math. Phys. $126(2)$ (1989), 325-346.

[36] M. Wang, Parallel spinors and parallel forms, Ann. Global Anal. Geom. 7 (1) (1989), 59-68.

[37] M. Wang, Preserving parallel spinors under metric deformations, Indiana Univ. Math. J. 40 (3) (1991), 815-844.

[38] R. Ye, Ricci flow, Einstein metrics and space forms, Trans. Amer. Math. 338 (2) (1993), 871-896.

Mathematics Department

UCSB

SANTA BARBARA

CA 93106

USA

E-mail address: dai@math.ucsb.edu

E-mail address: wei@math.ucsb.edu

Mathematics Department

Michigan State University

EAST LANSING

MI 48824

USA

E-mail address: xwang@math.msu.edu

Received December 21, 2005 